\author{
EESTI NSV TEADUSTE AKADEEMIA TOIMETISED, 32. KOIDE \\ FOUSIKA * MATEMAATIKA, 1983, NR. 3 \\ ИЗВЕСТИЯ АКАДЕМИИ НАУК ЭСТОНСКОИ ССР. ТОМ 32 \\ ФИЗИКА * МАТЕМАТИКА. 1983, № 3
}

удК $62-501.12$

Ю. НУРГЕС

\title{
МОДИФИКАЦИЯ ЧАСТИЧНОЙ РЕАЛИЗАЦИИ ДИСКРЕТНОЙ СИСТЕМЫ РАЗЛОЖЕНИЕМ ЛАГЕРРА
}

D. NURGES. DISKREETSE SOSTEEMI OSALISE REALISATSIOONI MODIFITSEERIMINE LAGUERRE'I TEISENDUSEGA

D. NURGES. MODIFICATION OF PARTIAL REALIZATION OF DISCRETE-TIME SYSTEMS BY LAGUERRE EXPANSION

\section{(Представил Н. Алумяэ)}

Рассмотрим дискретную динамическую систему

$$
\begin{aligned}
x(t+1) & =F x(t)+G u(t), \\
y(t) & =H x(t), \quad t=0,1,2, \ldots,
\end{aligned}
$$

где $x-n$-вектор состояния, $u-m$-вектор управляющих воздействий, $y$ - $p$-вектор выходных переменных. Матрицы $F, G, H$ имеют размерность $n \times n, n \times m$ и $p \times m$ соответственно.

Задача реализации заключается в отыскании тройки матриц $(F, G, H)$ по заданным марковским параметрам системы $M(i)$, $i=1,2, \ldots$

$$
M(i)=H F^{i-1} G
$$

т. е. в определении модели состояния системы (1), (2) по ее вход-выход соотношению. При конечном числе марковских параметров $M(i), i=$ $=1, \ldots, N$ решается задача частичной реализации $\left[{ }^{1}\right]$. Если $N \geqslant 2 n$, то решения обеих задач совпадают. А если $N<2 n$, частичная реализация дает некоторую аппроксимацию системы.

Полноценным' (представлением ұустойчивой динамической системы (1), (2) является ее лагеррова модель [2]:

$$
\begin{aligned}
z_{k+1} & =A z_{k}+B u_{k}, \\
y_{k} & =C z_{k}+D u_{k}, \quad k=0,1,2, \ldots,
\end{aligned}
$$

где $u_{k}$ и $y_{k}$ - векторы коэффициентов разложения переменных $u(t)$ и $y(t)$ по разностным многочленам Лагерра $\Psi_{k}(t)$

$$
\Psi_{k}(t)=\sqrt{1-\xi^{2}} \sum_{j=0}^{k}(-1)^{k+j}\left(\begin{array}{c}
k \\
j
\end{array}\right)\left(\begin{array}{c}
t+k-j \\
k
\end{array}\right) \xi^{t+k-2 j}
$$

$\xi \in[0,1),\left(\begin{array}{l}k \\ j\end{array}\right)-$ биномиальный коэффициент, 


$$
\begin{aligned}
& u_{k}=\sum_{t=0}^{\infty} u(t) \Psi_{k}(t), \\
& y_{k}=\sum_{t=0}^{\infty} y(t) \Psi_{k}(t),
\end{aligned}
$$

a

$$
\begin{gathered}
z_{k}=(I-\xi F) x_{k}-\xi G u_{k}, \\
A=(F-\xi I)(I-\xi F)^{-1}, \\
B=\left(1-\xi^{2}\right)(I-\xi F)^{-1} G, \\
C=H(I-\xi F)^{-1}, \\
D=H(I-\xi F)^{-1} G .
\end{gathered}
$$

Марковские параметры лагерровой модели (4), (5)

$$
\begin{aligned}
& M_{0}=D, \\
& M_{i}=C A^{i-1} B, \quad i=1,2, \ldots ?
\end{aligned}
$$

легко определить по весовой функции системы $h(t)\left[{ }^{2}\right]$

$$
\begin{gathered}
M_{i}=\left[m_{i}(\alpha \beta)\right], \quad \alpha=1, \ldots, p, \quad \beta=1, \ldots, m, \\
m_{i}=\Psi_{0}^{-1}(0)\left(h_{i}-\xi h_{i-1}\right), \\
h_{i}=\sum_{t=0}^{\infty} h(t) \Psi_{i}(t) .
\end{gathered}
$$

Оказывается, что можно комбинировать марковские параметры системы $M(i)$ и ее лагерровые модели $M_{j}$ для решения задач реализации.

Обозначим

$$
\bar{M}_{i}=H(I-\xi F)^{i-1} G .
$$

Тогда на основании определения (3)

$$
\bar{M}_{i}=\sum_{j=0}^{i-1}(-\xi)^{j}\left(\begin{array}{c}
i-1 \\
j
\end{array}\right) M(j), \quad i=1,2, \ldots,
$$

а из формул $(6)-(11)$, учитывая перестановочность матриц $F-\xi I$ и $I-\xi F$, получим

$$
\begin{aligned}
& M_{0}=\xi \bar{M}_{0}, \\
& M_{i}=\xi^{-i} \sum_{j=0}^{i-1}(-1)^{j}\left(1-\xi^{2}\right)^{j+1}\left(\begin{array}{c}
i-1 \\
j
\end{array}\right) \bar{M}_{-j-1}, \quad i=1,2, \ldots
\end{aligned}
$$

Матрицы $\bar{M}_{i}, i=-L,-L+1, \ldots, 0,1, \ldots$ представляют собой марковские параметры системы $(\bar{A}, \bar{B}, \bar{C})$, где

$$
\begin{aligned}
& \bar{A}=I-\xi F, \\
& \bar{B}=(I-\xi F)^{-L-1} G, \\
& \bar{C}=H .
\end{aligned}
$$

Тройку матриц $(\bar{A}, \bar{B}, \bar{C})$ можно определить решением задачи частичной реализации порядка $N$ по последовательности $\bar{M}_{-L}, \ldots, \bar{M}_{0}, \ldots$ $\ldots, \bar{M}_{N-L-1}$, а систему $(F, G, H)$ - из соотношений $(12)-(14)$ 


$$
\begin{aligned}
& F=\xi^{-1}(I-\bar{A}), \\
& G=\bar{A}^{L+1} \bar{B}, \\
& H=\bar{C} .
\end{aligned}
$$

Полученная частичная реализация системы (1), (2) имеет следующие отличия от обыкновенной частичной реализации порядка $N$ :

a) точно реализуется $N-L-1$ марковских параметров системы и $L+1$ параметров ее лагерровой модели;

б) свойства частичной реализации можно варьировать с выбором числа $L$ и постоянной $\xi$.

Отметим некоторые частные случаи модифицированной частичной реализации:

1. Если $L=1$, то получаем обыкновенную частичную реализацию порядка $N$.

2. Если $L=N$, то получаемая частичная реализация совпадает с частичной реализацией порядка $N$, определенной по лагерровой модели системы [2].

3. Если $L=0, \xi=1$, то получаем частичную реализацию без статической ошибки, так как

$$
M_{0}=H(I-F)^{-1} G=\sum_{i=1}^{\infty} M(i)=S(\infty),
$$

где $S(\infty)$ - матрица конечных значений переходных характеристик системы (1), (2).

Итак, предложенная модифицированная частичная реализация включает как обыкновенную частичную реализацию, так и частичную реализацию по лагерровой модели, а также их всевозможные комбинации. Последнее обстоятельство является особенно ценным тогда, когда обыкновенная частичная реализация не удовлетворяет предъявляемым требованиям (напр., оказывается неустойчивой).

\section{ЛИТЕРАТ У РА}

1. Tether, A. J. IEEE Trans. Automat. Contr., 15, № 4, 427 (1970),

2. Нургес Ю., Яаксоо Ю. Изв. АН ЭССР, Физ. Матем., 30, № 3, 209-219 (1981).

Институт кибернетики Академии наук Эстонской ССР
Поступила в редакцию 21/XII 1982 